\title{
LA PROSPECTIVA TECNOLÓGICA COMO UNA CAPACIDAD DINÁMICA EN LOS CENTROS DE INVESTIGACIÓN. CASO DE ESTUDIO.
}

\author{
TECHNOLOGICAL FORESIGHT AS A DYNAMIC CAPABILITY IN RESEARCH \\ CENTERS. CASE STUDY.
}

\author{
DOI: www.doi.org/10.54198/innova08.05 \\ Carla Patricia Bermúdez Peña \\ (D) Francisco Flores Agüero².
}

Cómo citar esté artículo: Bermúdez Peña, C. P., \& Flores Agüero, F. (2021). La prospectiva tecnológica como una capacidad dinámica en los centros de investigación. Caso de estudio, Revista Innova ITFIP, 8 (1), 48-66

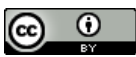

Recibido: febrero de 2021. Aprobado: junio de 2021

\section{Resumen}

En la actualidad, las organizaciones deben de estar preparadas para los cambios tecnológicos que les presenta su entorno y anticiparse a estos cambios para ajustar su estrategia tecnológica, esto se logra a través de la prospectiva tecnológica. El presente trabajo tiene como objetivo analizar como la prospectiva tecnológica puede ser una capacidad dinámica de una organización, específicamente de un centro de investigación. Para la presente investigación se tomó como caso de estudio el área de electroquímica del Centro de Investigación y Desarrollo Tecnológico en Electroquímica (CIDETEQ). Se realizaron entrevistas semiestructuradas a los actores principales encargados de la prospectiva tecnológica. Se identifican cinco características en común entre las capacidades dinámicas y la prospectiva tecnológica, estas características son: son una capacidad construida y no comprada, integran recursos, reconfiguran recursos, ganan recursos y permiten a las organizaciones adaptarse y crear cambios en el entorno. Se concluye que la prospectiva tecnológica

\footnotetext{
${ }^{1}$ Doctora en Gestión de la Tecnología, Facultad de Contaduría y Administración, Universidad Autónoma de Querétaro, Querétaro, México. ORCID: https://orcid.org/0000-0003-4728-7080, carla.bermudez@uaq.mx

2* Doctor en Gestión de la Tecnología, Facultad de Contaduría y Administración, Universidad Autónoma de Querétaro, Querétaro, México. ORCID: https://orcid.org/0000-0002-6708-8401 ,francisco.flores@uaq.mx
} 
REVISTA INNOVA ITFIP, 8 (1). 48-66. JUN. 2021

Es una capacidad dinámica construida capaz de integrar, reconfigurar y ganar recursos que permite adaptarse al entorno de una organización.

Palabras Clave: Capacidades dinámicas, Prospectiva tecnológica, Entorno dinámico, Centros de investigación.

\begin{abstract}
Currently, organizations must be prepared for the technological changes presented by their environment and anticipate these changes to adjust their technological strategy, this is achieved through technological foresight. The present work aims to analyze how technological foresight can be a dynamic capacity of an organization, specifically a research center. For the present investigation, the electrochemistry area of the Center for Research and Technological Development in Electrochemistry (CIDETEQ) was taken as a case study. Semi-structured interviews were carried out with the main actors in charge of technological foresight. Five characteristics are identified in common between dynamic capabilities and technological foresight. These characteristics are: they are a built and not purchased capacity,
\end{abstract}

Keywords: Dynamic capabilities, Technological foresight, Dynamic environment, Research centers.

\title{
Introducción
}

Actualmente las organizaciones se encuentran dentro de un entorno dinámico donde los altos niveles de competencia, el constante cambio de la demanda así como la obsolescencia de las tecnologías, suceden rápidamente, provocando de ésta manera que la información al alcance de las organizaciones no siempre sea exacta, certera e incluso, en muchos casos, no se encuentra disponible (Eisenhardt, 1989). Existen diversos enfoques estratégicos que afirman que la toma de decisiones dentro de estos contextos se ve alterada por: 1) el largo proceso de recolección de datos, así como por el afán de comprender en su totalidad la información obtenida; o su contraparte, el considerar pocas alternativas y obtener información de pocas fuentes. 2) La participación limitada y el poder centralizado de la administración y/o 3) el conflicto entre los administradores de la organización, creando interrupción en el proceso de toma de decisiones. Sin embargo, existe evidencia que el cambio organización se caracteriza por largos períodos de cambio lento e incremental y breves períodos de cambio rápido discontinuo o radical (Brown \& Eisenhardt, 1997).

Cómo respuesta a la necesidad constante de adaptación a los entornos dinámicos, la anticipación a necesidades futuras del mercado, desarrollo tecnológico como tendencias, que permita ayudar a la sobrevivencia de las empresas a largo plazo como su éxito económico, surge la prospectiva tecnológica (Reger, 2001). De acuerdo al nivel de cambio que enfrente la organización, puede ser 
definida como un proceso o una capacidad (Rohrbeck, 2010), motivo por el cuál dentro de la literatura no existe un consenso de una definición o concepto único. No obstante, cabe destacar que ésta amplia variedad de términos incluye diferentes actividades dentro de la empresa, de acuerdo al enfoque de estudio.

Reger (2001) define la prospectiva tecnológica como la exploración sistemática y la observación de las nuevas tecnologías o las tecnologías existentes, la evaluación de su potencial y su importancia para la competitividad de la empresa, así como el almacenamiento y la difusión de la información, incluyendo análisis, búsqueda, monitoreo y pronóstico de la tecnología. El enfoque que utiliza es de un proceso que va desde el monitoreo tecnológico, la vigilancia tecnológica, la previsión tecnológica, la exploración tecnológica y la evaluación de la tecnología, entre otras.

Sin embargo, desde un enfoque holístico de cambios tecnológicos, ambientales y estructurales dentro de la organización, la prospectiva tecnológica en la organización puede ser una capacidad que incluye cualquier mecanismo estructural o cultural que permita a la empresa descubrir cambios discontinuos, interpretar los resultados y precisar respuestas objetivas que aseguren la supervivencia de la misma (Rohrbeck, 2010).

El objetivo del presente trabajo es relacionar el concepto de prospectiva tecnológica con las capacidades dinámicas dentro de los centros de investigación, considerando a las capacidades dinámicas como las rutinas organizativas y estratégicas con las cuáles las empresas logran nuevas configuraciones de recursos a medida que los mercados surgen, chocan, se dividen, evolucionan y mueren (Eisenhardt \& Martin, 2000). Al poder relacionar estos dos conceptos, la prospectiva tecnológica pasará a formar parte de las actividades esenciales de una organización y no solo ser vista como una actividad complementaria. Esto permitirá a las organizaciones estar mejor preparadas para los cambios en el mercado.

Por lo anterior, la prospectiva tecnológica puede ser considerada como una capacidad dinámica que permita integrar, reconfigurar, obtener y liberar recursos, para igualar e incluso crear cambios en el mercado. 


\section{Metodología}

Para la presente investigación se reconoce la necesidad de encontrar una organización que desarrolle la actividad de prospectiva tecnológica. Se identifica al Centro de Investigación y Desarrollo Tecnológico en Electroquímica (CIDETEQ) como una organización que ha desarrollado prospectiva tecnológica por los últimos 4 años. El CIDETEQ es líder en el país en el desarrollo e investigación en tecnología electroquímica. También proporciona diferentes servicios a la industria, como análisis de metales, análisis de aguas, caracterización de materiales y análisis de fallas. Dentro de sus objetivos estratégicos se encuentra desarrollar transferir paquetes tecnológicos propios.

En el acercamiento inicial con la organización, se habla con el Director de Planeación e Inteligencia Tecnológica en donde se conocen las características esenciales de la organización y como se desarrolla la prospectiva tecnológica dentro de esta. Posteriormente se realizaron entrevistas semiestructuradas con los principales actores. Se utilizaron entrevistas semiestructuradas ya que es una herramienta flexible para investigaciones con un número de participante bajo. Las entrevistas semiestructuradas se utilizan cuando la investigación se beneficiaría de un marco bastante abierto. También se usan cuando se puede obtener información más útil de una comunicación bidireccional enfocada pero conversacional con los participantes (Drever, 1995). Para preparar la entrevista únicamente se identificaron temas y subtemas del tema a investigar. Las preguntas específicas surgieron en el curso de la entrevista al momento de abordar los temas y subtemas.

El tema principal abordado fueron las características y condiciones bajo las cuales se realiza la prospectiva tecnológica en el Centro de Investigación. La entrevista fue dirigida para conocer si la prospectiva tecnológica empataba con las características brindadas por Eisenhardt and Martin (2000). De igual forma se realizaron preguntas para indagar si la actividad de prospectiva tecnológica podía categorizarse como capacidad dinámica de acuerdo a la tipología propuesta por Teece (2007). Los resultados más relevantes se presentan a continuación.

\section{Marco Teórico}

\section{Prospectiva tecnológica}

La prospectiva tecnológica moderna tiene sus raíces en las agencias gubernamentales de Estados Unidos, especialmente en el departamento de defensa entre los años de 1950 y 1960. Los estudios de prospectiva realizados por las agencias de Estados Unidos tenían como objetivo ayudar a dirigir de manera correcta las inversiones en tecnología (Ende, Mulder, Knot, Moors, \& Vergragt, 1998). En la década de los 70's varias agencias gubernamentales de Japón comenzaron a conducir estudios de prospectiva tecnológica (Martin, 1995). Mientras que en la década de los noventa los países de Europa comenzaron a utilizar la prospectiva tecnológica de manera sistemática (Blind, Cuhls, \& Grupp, 1999). En el caso de la prospectiva tecnológica en las organizaciones, los primeros estudios fueron conducidos en Inglaterra en la universidad de Sussex dentro del departamento de Science Policy Research Unit (SPRU) entre 1970 y 1980 (Miles, 2010). 
En la literatura existen dos enfoques importantes con los cuales se estudia a la prospectiva tecnológica. El primero enfoque es el estudio de la prospectiva tecnológica como un proceso (Becker, 2002; Horton, 1999; Reger, 2001; Will, 2008), mientras que el segundo enfoque estudia a la prospectiva como una habilidad (MacKay \& McKiernan, 2004; Rohrbeck, 2010; Slaughter, 1998). Para la presente investigación se estudiará a la prospectiva como una habilidad y se retomará la definición de Rohrbeck (2010) que define a la prospectiva tecnológica como la habilidad que tiene una organización para detectar cambios discontinuos de forma temprana, interpretar sus conciencias y formular respuestas efectivas que ayuden a la supervivencia de la empresa a largo plazo.

El objetivo principal de la prospectiva tecnológica es apoyar los nuevos desarrollos en las áreas de ciencia y tecnología, reconociendo con prontitud los beneficios de éstos desarrollos ya sea para una nación o una organización (Becker, 2002). Aunque la prospectiva tecnológica en las organizaciones comparte este objetivo en común, de acuerdo a Martin (1995) las empresas normalmente se centran en una de estas funciones intermedias:

1) Inteligencia anticipatoria. La prospectiva provee antecedentes, consejos generales futuros y alertas tempranas de los desarrollos más recientes.

2) Ajuste de dirección (Direction-setting). Se establecen lineamientos generales para la estrategia corporativa, pero sin un involucramiento directo en la toma de decisiones.

3) Determinación de prioridades. La prospectiva sirve para identificar las líneas de I+D en donde la organización deberá invertir.

4) Formulación de estrategias e implementación. La prospectiva es utilizada como parte integral en la formulación e implementación de estrategias. En este caso comúnmente las personas encargadas de realizar la prospectiva son las encargadas de formular las estrategias.

\section{La prospectiva tecnológica dentro de las organizaciones}

En las organizaciones la prospectiva tecnológica no es una actividad estructurada y sistemática, por lo tanto, no existen actividades plenamente definidas que en su conjunto construyan el concepto de prospectiva tecnológica. Sin embargo, Reger (2001) logró identificar algunas fases comunes entre las diferentes organizaciones.

Determinación de las necesidades de información y selección del área de búsqueda. Esta fase se puede abordar por medio de dos perspectivas. La primera es la perspectiva "inside-out" en dónde se delimita el área de observación de acuerdo al dominio tecnológico de la empresa. La segunda perspectiva se denomina "outside-in" en donde no se delimita el área de observación y se busca cualquier tecnología potencial que sirva a la organización, posteriormente se evalúan los resultados de acuerdo a su relevancia. 
Selección de las fuentes de información. En este sentido existen dos grandes fuentes de información en las cuales las organizaciones pueden encontrar datos para realizar prospectiva tecnológica: información formal e informal. La información formal está documentada y puede ser obtenida a pesar de las distancias geográficas, mientras que la información informal se no se encuentra escrita y se transmite vía oral. Las fuentes de información formal son: Journals científicos, revistas, reportes, estudio de tendencias, internet etc. Las fuentes de información informal son: Congresos, contactos personales con redes externas, contratación de los mejores recursos humanos en un área en específico y trabajo en redes con clientes y proveedores.

Filtrar, analizar e interpretar la información. La información recolectada debe de ser filtrada, analizada e interpretada antes de tomar una decisión, donde todos los actores involucrados deben de tomar parte en esta fase. Para realizar el filtrado de la información se debe determinar cuál es información relevante y cuál no; una vez determinada, la información de relevancia es almacenada en las bases de datos de la organización para posteriormente discutir las posibles implicaciones que tienen esta información con las actividades de la organización.

Toma de decisiones. El objetivo final de la prospectiva tecnológica es influir y apoyar las decisiones administrativas respecto a los recursos que se destinarán a investigación y tecnología. Esencialmente la prospectiva tecnológica puede ayudar a una organización a: 1) crear nuevos proyectos o modificar los ya existentes, 2) crear nuevos programas de investigación o modificar los ya existentes 3 ) crear proyectos estratégicos de innovación interdepartamentales, 4) conocer nuevos campos de innovación y 5) establecer las prioridades del departamento de I+D.

\section{La prospectiva tecnológica bajo la teoría basada en recursos (Resource-Based View)}

La teoría basada en recursos se fundamenta en el concepto central de que la habilidad que tienen las empresas para competir exitosamente contra sus rivales depende de ciertos recursos estratégicos de la empresa (Wernerfelt, 1984). Para que estos recursos puedan generar una ventaja competitiva deben de ser: 1) valiosos, 2) raros y escasos, 3) no imitables y 4) no sustituibles (Eisenhardt \& Martin, 2000). Al usar estos recursos las empresas pueden crear una ventaja competitiva sostenible creando estrategias que los competidores no pueden imitar de manera fácil (Barney, 1991).

En sentido, la prospectiva tecnológica es un recurso que permite a las organizaciones explorar y explotar nuevas oportunidades de negocios antes que sus competidores que carecen de este recurso. Por esta razón se puede calificar a la prospectiva tecnológica como un recurso valioso (Schwarz, Rohrbeck, \& Wach, 2019). De igual forma los sistemas de prospectiva tecnológica son raros ya que su implementación no es una tarea fácil y pocas empresas logran tener éxito al ponerlo en funcionamiento. También se puede decir que la prospectiva no es imitable ya que depende de las características de cada organización, así como las fuentes de información con las que cuente. Finalmente se afirma que es un recursos insustituible ya que otros mecanismos fallan al momento de responder a los cambios en el ambiente de la organización (Rohrbeck, 2010). 
REVISTA INNOVA ITFIP, 8 (1). 48-66. JUN. 2021

\section{Perspectiva de las capacidades dinámicas}

El enfoque basado en recursos (RBV por sus siglas en inglés) propone que la ventaja competitiva se obtiene principalmente por contar con recursos valiosos, raros, inimitables y no sustituibles. El RBV ayuda a explicar de una manera general como una empresa logra obtener una ventaja competitiva. Sin embargo la teoría de este enfoque no es capaz de explicar en detalle como una empresa puede obtener una ventaja competitiva en un ambiente dinámico (Eisenhardt \& Martin, 2000). Debido a que los recursos están basados en el contexto de las organizaciones, su valor depende de las características del entorno. Sin embargo, debido a que los recursos son menos dinámicos que el entorno, es común que estos queden rezagados en comparación con el entorno de la organización (Teece, 2017; Teece, Pisano, \& Shuen, 1997)

Para poder explicar cómo lograr una ventaja competitiva en un ambiente dinámico, los académicos han complementado el RBV con la perspectiva de la capacidad dinámica, destacando el papel de las capacidades para integrar, construir y reconfigurar las competencias internas y externas (Teece, 2017). Desde esta perspectiva, las empresas deben adaptar, integrar y reconfigurar constantemente sus recursos y competencias para responder de manera rápida a un entorno dinámico. Sin embargo, los procesos y rutinas organizacionales arraigadas, desarrolladas a partir de trayectorias anteriores o de la trayectoria de asignación de recursos y desarrollo de competencias, limitan esos cambios o adaptaciones (Teece et al., 1997). De igual forma, Teece et al. (1997) asumen que las capacidades dinámicas generalmente se construyen en lugar de comprarse y que su creación y su evolución están integradas en procesos organizacionales que están determinados por las posiciones de los activos de las empresas y los caminos evolutivos que han adoptado en el pasado.

Otros estudios desarrollan y clarifican los conceptos de capacidades dinámicas. Eisenhardt and Martin (2000), definen a las capacidades dinámicas como los procesos que una empresa utiliza (específicamente el proceso para integrar, reconfigurar, ganar y liberar recursos) para alcanzar e inclusos crear cambios en el mercado; por lo tanto, las capacidades dinámicas son las rutinas organizacionales y estratégicas por las cuales una empresa logra configurar nuevos recursos mientras los mercados emergen, colisionan, se separan, evolucionan y mueren. Además de aportar una definición de capacidades dinámicas, Eisenhardt and Martin (2000) afirman que las capacidades dinámicas difieren de acuerdo al dinamismo del mercado. En mercados con una dinámica moderada las capacidades dinámicas tienden a ser rutinas eficientes y procesos robustos. Por otra parte, en los mercados con una alta velocidad de cambio las capacidades dinámicas dependen en mayor medida del conocimiento desarrollado en situaciones específicas. Por lo tanto, en los mercados con una alta velocidad, se hace indispensable poder tener información acerca de las posibles alternativas en las que pueden desembocar los cambios.

Por su parte, Zollo and Winter (2002) definen a las capacidades dinámicas como un patrón aprendido y estable de actividades colectivas, mediante los cuales una organización genera y modifica sistemáticamente sus rutinas operativas para mejorar su efectividad. Además, se da un gran énfasis en el conocimiento para poder crear las capacidades dinámicas de una empresa por 
medio de la acumulación de experiencia, articulación del conocimiento y el proceso de codificación del conocimiento. La acumulación de experiencia se da cuando un conjunto de patrones estables llamados rutinas comienzan a crear conocimiento tácito que se pude utilizar posteriormente para responder a cambios en el entorno. La articulación del conocimiento se refiere a la forma en la cual la organización logra convertir las experiencias individuales de sus miembros en conocimiento que ayude a mejorar los resultados de la organización. Por último, la codificación del cocimiento se consigue cuando el conocimiento articulado se logra plasmar en una herramienta que facilite su utilización o difusión.

\section{Tipos de capacidades dinámicas}

Teece (2007), amplía su definición de capacidades dinámicas argumentando que estas se pueden separar en: 1) capacidad para detectar y dar forma a oportunidades y amenazas (sensing), 2) para aprovechar las oportunidades (seizing) y 3) para mantener la competitividad mejorando, combinando, protegiendo $\mathrm{y}$, cuando sea necesario, reconfigurando los activos intangibles $\mathrm{y}$ tangibles de la empresa comercial (transforming). Posteriormente estas capacidades fueron categorizadas como capacidades dinámicas de alto nivel, lo que significa que ayudan a la organización a conocer posibles vertientes sobre el futuro, diseñar modelos de negocios para aprovechar nuevas oportunidades y determinar la mejor configuración para la organización de acuerdo a los planes futuros (Teece, 2017). En la Figura 1 se resumen estas capacidades.

Figura 1. Esquema simplificado de las capacidades dinámicas

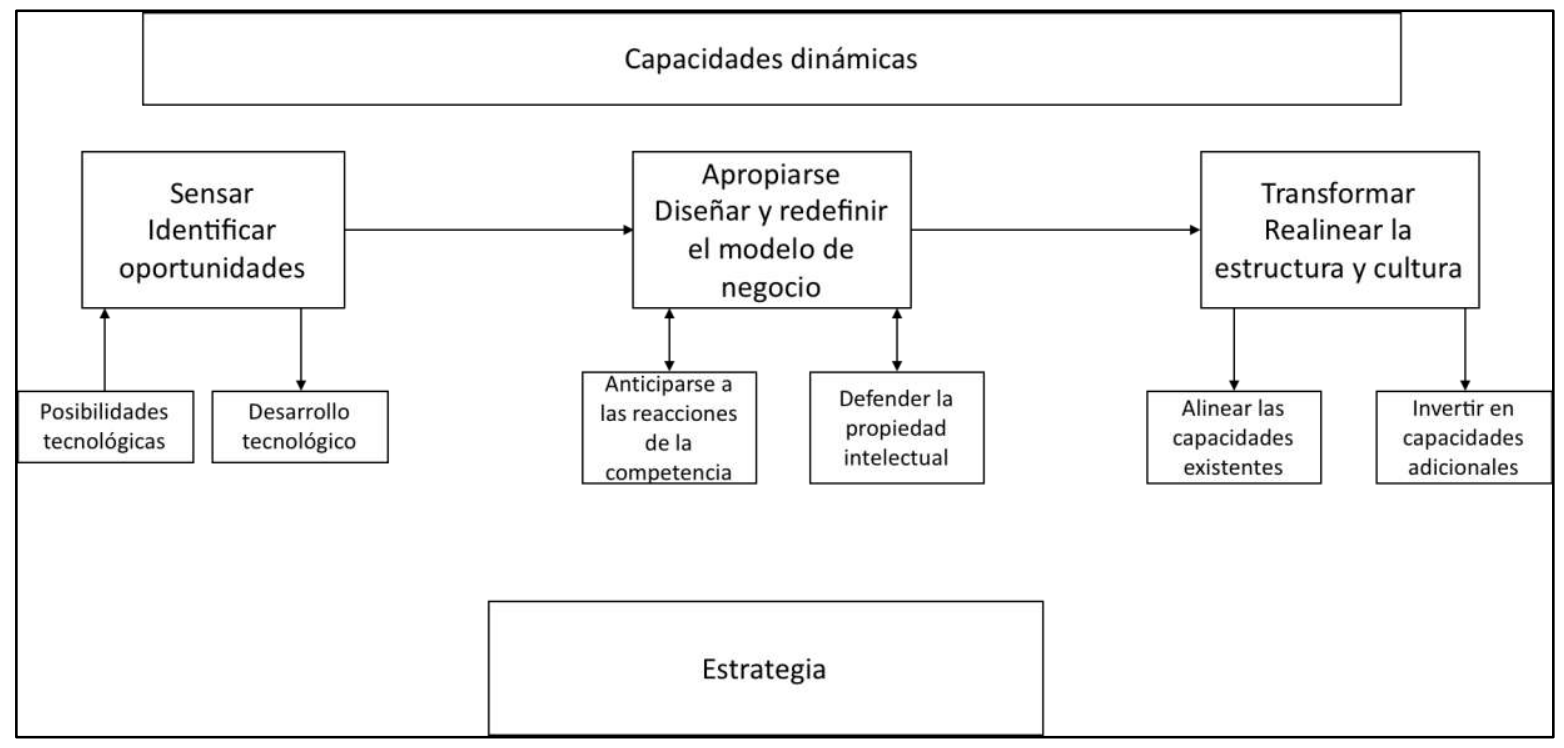

Fuente: (Teece, 2017) 
REVISTA INNOVA ITFIP, 8 (1). 48-66. JUN. 2021

Capacidad para detectar oportunidades (sensing). De acuerdo a Teece (2007) la capacidad de detectar las nuevas oportunidades está formada por las actividades de escanear, crear, aprender e interpretar. La capacidad de encontrar o crear una nueva oportunidad proviene de los de la capacidades cognitivas y creativas de los individuos de una organización. Sin embargo, está capacidad también puede estar fincada en los procesos organizacionales tales como las actividades de investigación o desarrollo. Esta capacidad también necesita del componente de interpretación para poder configurar la información que se obtiene, así como los de escanear y monitorear los desarrollos tecnológicos internos y externos. Las actividades de búsqueda que son relevantes para detectar nuevas oportunidades incluyen información sobre lo que está sucediendo en el ecosistema de negocios. Concluyendo, la capacidad para detectar nuevas oportunidades es un sistema analítico para aprender, filtrar, dar forma y calibrar oportunidades.

Capacidad para aprovechar oportunidades (seizing). Una vez que una oportunidad tecnológica o de mercado ha sido detectada ésta debe abordarse a través de nuevos productos, procesos o servicios. Esta capacidad se fundamenta en la selección de las arquitecturas del producto y modelos de negocio. Este tipo de capacidad abarca: 1) qué tecnologías y características tienen que ser integradas al nuevo producto o servicio, 2) cómo dese ser diseñada la estructura de ingresos y costos para satisfacer las necesidades de los clientes, 3) la forma en que las nuevas tecnologías deben de ser ensambladas, 4) identificar los segmentos de mercado a los que irá dirigido el nuevo producto o servicio y 5) el mecanismo por el cual el valor será capturado. Esta capacidad tiene que crear, ajustar, perfeccionar y si es necesario reemplazar los modelos comerciales existentes de la empresa.

Capacidad para mantener la competitividad (transforming). Una vez que una organización ha logrado identificar y aprovechar las oportunidades se puede alcanzar el crecimiento y la rentabilidad de esa organización. Este crecimiento conducirá al incremento de los recursos y activos de la organización, la clave para poder sostener este crecimiento está en la habilidad para recombinar y reconfigurar los bienes y estructuras organizacionales.

Como se ha podido observar, las capacidades dinámicas hacen referencia a la capacidad de las organizaciones para adaptarse ante un entorno dinámico. Para que la empresa pueda adaptarse en necesario saber que tendencias existen en los mercados para poder preparase para los cambios. Es en este punto en donde la prospectiva tecnológica puede ser vista como una capacidad dinámica ya que es una habilidad de la empresa para detectar cambio de forma temprana.

\section{Resultados y Discusión}

En la organización estudiada, el desarrollo de prospectiva tecnológica nace debido a la necesidad de los investigadores de conseguir proyectos de investigación que pudieran financiar a la organización. Se detecta que en el entorno en el cual se desenvuelve la organización es necesario que cuenten con un alto impacto de investigación para poder ser financiados tanto por el gobierno 
como por organizaciones privadas. La organización detecta que este impacto lo consigue estando a la vanguardia en investigación y desarrollos tecnológicos conseguida por medio de la prospectiva tecnológica.

Para que una capacidad de una organización sea dinámica, se identifican los siguientes factores clave: 1) es una capacidad construida y no comprada, 2) integra recursos, 3) reconfigura recursos, 4) gana recursos y 5) permitir adaptarse a un entorno dinámico o incluso crear cambios en este. Como ya se mencionó durante la revisión de literatura, una característica de la prospectiva tecnológica es que son sistemas difíciles de implementar en donde pocas empresas tienen éxito. La dificultad de la prospectiva tecnológica radica en que se necesita conjuntar conocimiento técnico acerca del tema abordado y una alta experiencia para poder dar sentido a la información que se obtiene. En el centro de investigación estudiado la prospectiva tecnológica tiene lugar hasta que un experto en inteligencia tecnológica con capacidad para interpretar la información se integra a la organización. Debido a la especificidad de los temas que desarrolla la organización se requiere un nivel alto de conocimientos técnicos, estos conocimientos técnicos son inherentes a los investigadores que tienen una amplia experiencia a nivel internacional. Por lo tanto, en el caso de la organización estudiada, la prospectiva tecnológica es el resultado del desarrollo de conocimiento técnico y de interpretación generado a través de varios años y que no puede ser comprado ni imitado, únicamente puede ser construido debido a las características propias de la organización.

Una de las características de la prospectiva tecnológica es crear proyectos de investigación interdepartamentales. En este mismo sentido, las capacidades dinámicas tienden a integrar recursos. En el centro de investigación, uno de los resultados de la prospectiva tecnológica fue integrar coordinaciones en subdirecciones. De tener siete coordinaciones, la organización pasa a tener 4 subdirecciones, atendiendo a las áreas estratégicas reconocidas en el ejercicio de prospectiva. La reducción de coordinaciones permitió a la organización enfocarse a áreas con gran potencial de crecimiento que les permitió desarrollar proyectos con un gran valor en el entorno de la organización.

Otro objetivo de las capacidades dinámicas es reconfigurar recursos existentes de la organización. De igual forma la prospectiva tecnológica permite modificar programas de investigación existentes de acuerdo a las necesidades identificadas por el ejercicio. En el caso del centro de investigación se detectó que una tendencia en los próximos años sería la utilización de materiales de adsorción para el tratamiento de agua. Por lo tanto, se tuvo que dar una reconfiguración de las capacidades del centro de investigación para poder abordar este tema.

Un objetivo final de la prospectiva tecnológica es crear nuevos proyectos líneas de investigación. Para crear nuevas líneas de investigación es necesario crear nuevos recursos que permitan sostener su creación. De la misma forma, un objetivo de las capacidades de innovación es crear recursos. Dentro del caso de estudio, la prospectiva tecnológica ayudó a identificar el área de bioelectrónica como un área de prioridad para el centro de investigación. Esto obligó a la empresa a crear un laboratorio integrado de bioelectroquímica para poder investigar esta línea. De la misma forma se tuvieron que crear recursos intangibles como una cátedra y conocimientos de ciencia básica acerca 
del tema. Otro resultado de la creación de la línea de investigación de la bioelectrónica fue el reconocimiento por parte del gobierno mexicano de que la bioelectrónica es un área con un gran potencial de desarrollo. Este reconocimiento tuvo como consecuencia un apoyo económico. Lo anterior está relacionado con la característica de las capacidades dinámicas relativas a ganar nuevos recursos.

El fin último de la prospectiva tecnológica, es permitir a las organizaciones explorar y explotar nuevas oportunidades de negocios antes que sus competidores que carecen de este recurso. Hablando de las capacidades dinámicas, uno de sus objetivos es permanecer o crear cambios en el mercado. Al explorar y explotar nuevas oportunidades de negocio, la prospectiva tecnológica permite a la organización anticiparse y estar preparados para los cambios en el entorno.

Para el caso el centro de investigación, han notado que la electroquímica y los desarrollos tecnológicos en estas áreas a nivel internacional, están dirigidos al estudio de materiales avanzados resistentes a la corrosión dirigida a la industria aeronáutica y automotriz. Como consecuencia se han preparado para abordar esta línea de investigación en los próximos años y de esta forma adaptarse a los cambios en el entorno. En la Tabla 1 se resumen las características de la prospectiva tecnológica que permiten verla como una capacidad dinámica.

Tabla 1. Características que permiten a la prospectiva tecnológica ser una capacidad dinámica

\begin{tabular}{l|l}
\hline Características de las capacidades dinámicas & Características de la prospectiva tecnológica \\
\hline Capacidad construida y no comprada & $\begin{array}{l}\text { Los conocimientos para la prospectiva son } \\
\text { construidos a través de la experiencia }\end{array}$ \\
\hline Integra recursos & $\begin{array}{l}\text { Integra proyectos de investigación y desarrollo } \\
\text { interdepartamentales de acuerdo a las tendencias } \\
\text { futuras detectadas. }\end{array}$ \\
\hline Reconfigura recursos & $\begin{array}{l}\text { Permite modificar programas de investigación } \\
\text { existentes de acuerdo a las necesidades } \\
\text { identificadas }\end{array}$ \\
\hline Ganar recursos & $\begin{array}{l}\text { Permite la atracción de nuevos recursos al } \\
\text { generar tecnologías y conocimiento nuevo }\end{array}$ \\
\hline Permite adaptarse y crear cambios en el entorno & $\begin{array}{l}\text { Permite conocer los cambios en el entorno antes } \\
\text { de que estos sucedan, lo que provoca que las } \\
\text { organizaciones estén preparadas para los } \\
\text { cambios. }\end{array}$ \\
\hline
\end{tabular}

Fuente: Elaboración propia. 
Como ya se ha mencionado durante el presente trabajo, la prospectiva tecnológica tiene como fin influir y apoyar las decisiones administrativas respecto a los recursos que se destinarán a investigación y tecnología. Para cumplir con su propósito, la prospectiva selecciona, filtra, analiza e interpreta información. Sin embargo, como ya se ha discutido en este trabajo, estas actividades pueden diferir o cambiar según la organización. De acuerdo a Teece (2007) la capacidad dinámica para detectar oportunidades (sensing) está conformada por las actividades de escanear, crear, aprender e interpretar. En el centro de investigación estudiado, la prospectiva tecnológica se desarrolla mediante las actividades de: 1) selección y filtración de la información, 2) análisis de la información e 3) interpretación de la información.

Para el caso del análisis de la información se pide a los investigadores dar una lista de palabras clave. Posteriormente por medio de diferentes herramientas electrónicas se hace una búsqueda de las palabras clave y se seleccionan aquellas que tengan más relevancia de acuerdo a número de apariciones (Figura 2).

Figura 2. Selección de información relevante

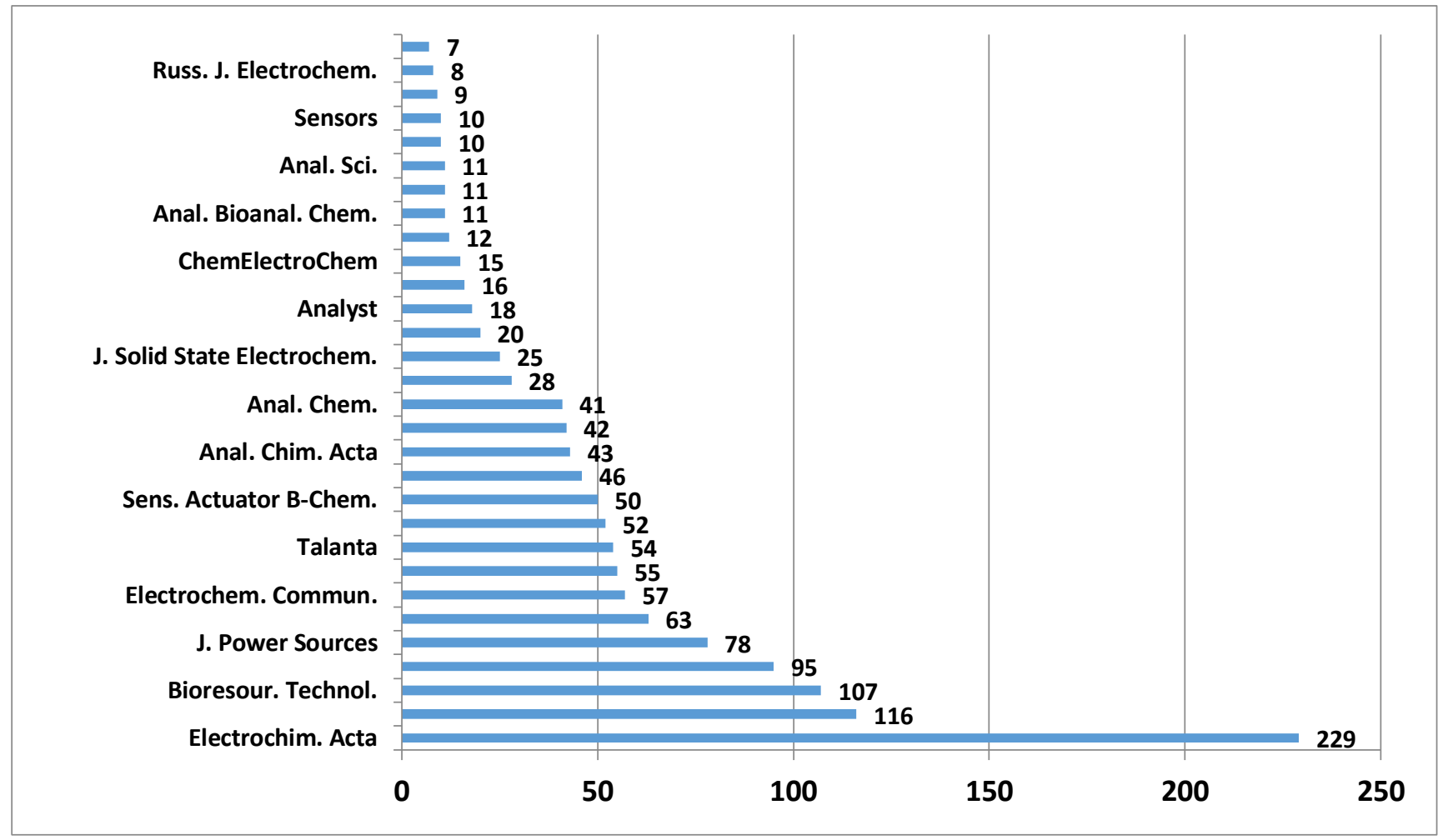

Fuente CIDETEQ (2015) 
REVISTA INNOVA ITFIP, 8 (1). 48-66. JUN. 2021

Posteriormente se hace un análisis de esa interpretación; por ejemplo, quienes son los investigadores a nivel mundial líderes en el tema, como están agrupados los núcleos de investigación y que impacto están teniendo estas investigaciones a nivel industria. Por último, se hace una interpretación acerca de las tendencias futuras y los cambios en los temas de investigación (Figura 3).

Figura 3. Mapa acerca de los cambios en los temas de investigación en el área de bioelectroquímica

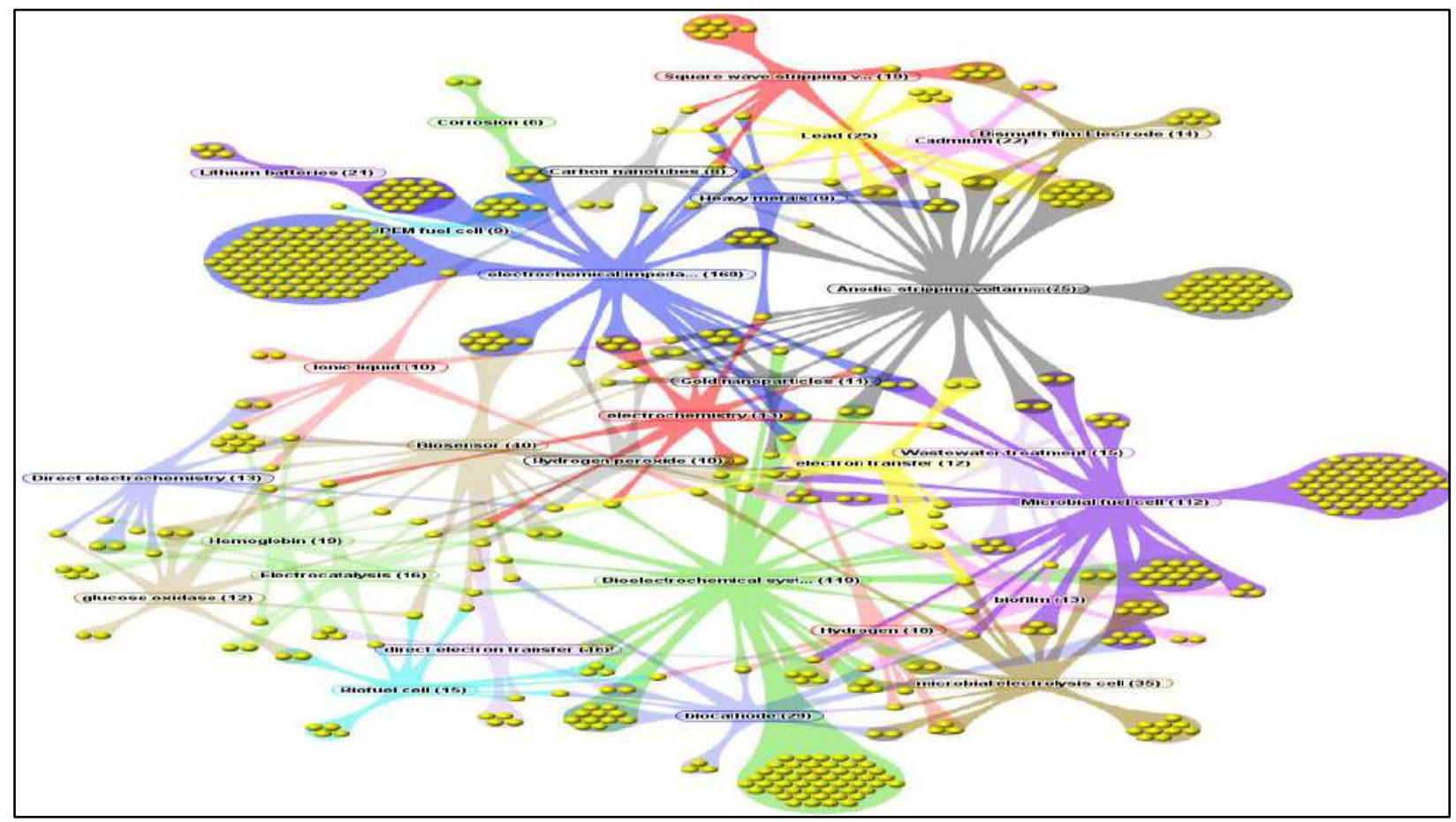

Fuente: CIDETEQ (2015)

Se puede apreciar que la prospectiva tecnológica, así como la capacidad dinámica para poder detectar oportunidades, comparten actividades y objetivos en común como se muestra en la figura 4. Por lo tanto, la prospectiva tecnológica es una capacidad dinámica para detectar oportunidades (sensing). 
Figura 4. Comparación entre la prospectiva tecnológica y la capacidad dinámica sensing

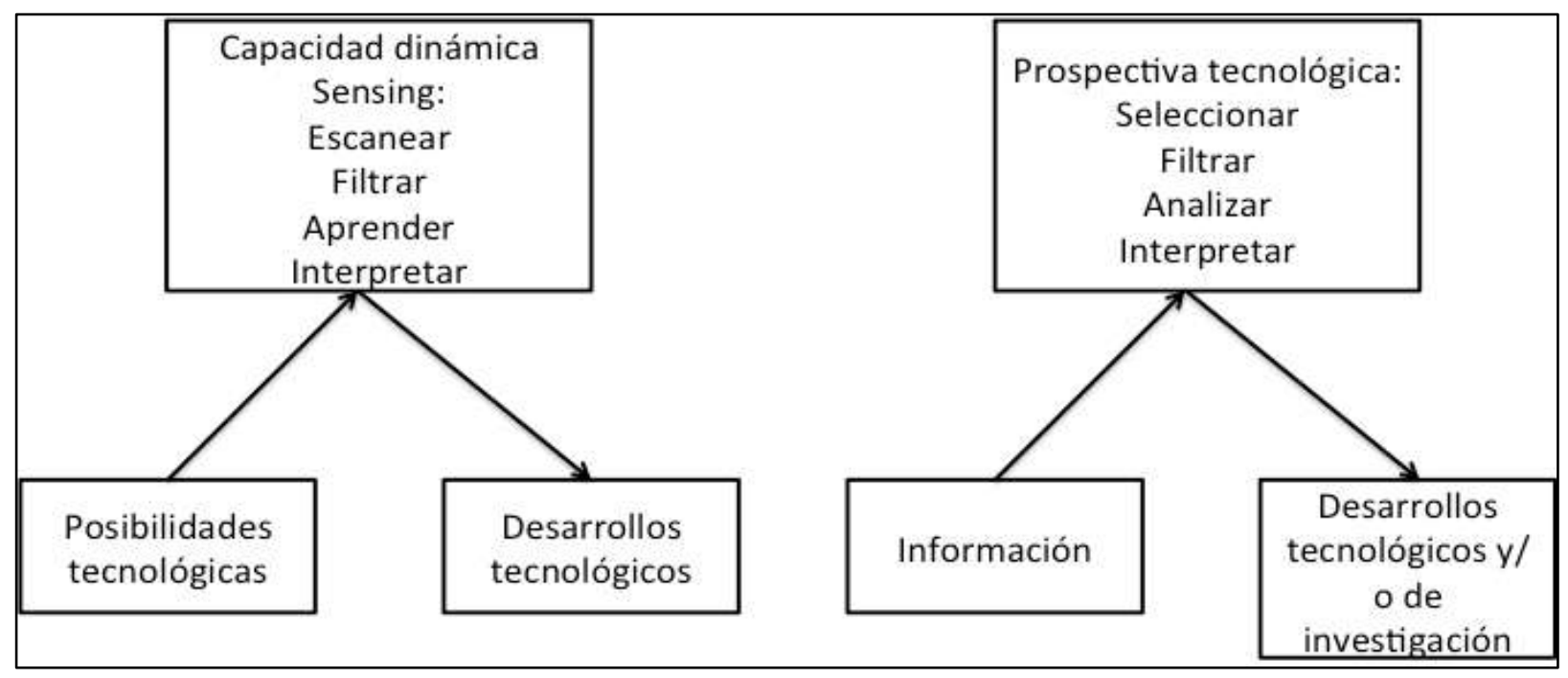

Fuente: Elaboración propia

\section{Discusión}

La prospectiva tecnológica es un concepto difícil de definir y de comprender, diferentes autores asignan diferentes conceptos a este término que va desde una habilidad (Ej. Rohrbeck, 2010) hasta una ciencia para dictar las políticas de innovación y tecnología de un país (Medina Vásquez \& Ortegón, 2006). Por el contrario, las capacidades dinámicas tienen un contexto claro dentro del campo de la administración estratégica (Miranda Torrez, 2015). Las capacidades dinámicas se pueden comparar con otras teorías de estrategia y se puede rastrear su desarrollo histórico; su ubicación en una disciplina académica y comercial bien establecida proporciona puntos de referencia claros para su estudio (Fallon-Byrne \& Harney, 2017). Estudiar a la prospectiva tecnológica como una capacidad central lo pone en un contexto, establecer relaciones entre prospectiva y estrategia hace que toda la literatura sobre estrategia esté disponible como contexto para el estudio de la prospectiva tecnológica.

De igual forma, el campo de la administración estratégica y las capacidades dinámicas se ve beneficiada al momento de relacionarlos con la prospectiva tecnológica, ya que al estudiar la prospectiva como una capacidad dinámica se muestra cómo se pueden identificar los elementos constitutivos de una capacidad dinámica a un nivel organizacional, así como explicar cómo se 
puede emplazar, nutrir y mantener una capacidad dinámica. El programa de prospectiva analizado en esta investigación muestra cómo de manera práctica un esquema coordinado de prospectiva tecnológica puede impactar en las competencias dinámicas de los centros de investigación, en donde estar informado de las nuevas tendencias tecnológicas y los nuevos campos de investigación generan una ventaja competitiva en este tipo de instituciones (Edington, 2001). Conectar la prospectiva a las capacidades centrales agrega un nuevo campo de estudio a la disciplina de la administración estratégica.

Una parte fundamental de las capacidades dinámicas es que se deben de encontrar integradas a la cultura organizacional (Bravo \& Herrera, 2019), esta es otra característica que comparten los conceptos de capacidades dinámicas y prospectiva tecnológica, ya que de acuerdo a Gutiérrez Encino y Gonzales Ospina (2018), para que en una organización se dé el proceso de prospectiva, este debe de estar presente en la cultura organizacional para que se pueda valorar el conocimiento y su uso de manera eficaz y eficiente.

Si bien Schwarz, Rohrbeck y Wach (2019) logran estudiar a la prospectiva tecnológica como un micro fundamento de las capacidades dinámicas, este se hace desde la perspectiva gerencial o de liderazgo de las empresas, el presente trabajo logra aterrizar algunos de los conceptos propuestos por estos autores como la integración de la prospectiva en las habilidades y tecnologías de la gerencia a un nivel operacional, en donde la implicación de ver a la prospectiva tecnológica como una capacidad dinámica a un nivel operacional ayuda a las organizaciones a crear conocimiento técnico, poder codificar este conocimiento, definir los roles de los miembros de la organización para utilizar este conocimiento y socializar dentro de la organización el conocimiento para que la prospectiva tecnológica forme parte de los valores y normas de la organización. Al igual que Teece (2017) expone que las capacidades dinámicas deben de integrar, reconfigurar y transformar recursos, la prospectiva tecnológica en el caso del centro de investigación estudiado logra penetrar en estos mismo aspectos, lo que provoca los miembros de esta institución reconozcan el valor agregado de la prospectiva tecnológica lo que en este caso en particular la eleva a una capacidad dinámica para el centro de investigación. Por último, este estudio encuentra que la prospectiva tecnológica al ser una capacidad dinámica para el centro de investigación estudiado se integra en las estrategias de esta organización para dictar las políticas de futuras investigaciones, esto concuerda con el trabajo de Pineda (2013) en donde se estudian a los grupos de investigación en universidades colombianas, y concuerda que el concepto de prospectiva tecnológica debe de integrarse a las políticas y estrategias de estos cuerpos de investigación, integrar la prospectiva tecnológica ya sea a centros de investigación o a grupos de investigación puede ayudar a mejorar las políticas y estrategias tecnológicas de un país. 
REVISTA INNOVA ITFIP, 8 (1). 48-66. JUN. 2021

\section{Conclusiones}

Se concluye que la prospectiva tecnológica es una capacidad dinámica construida capaz de integrar, reconfigurar y ganar recursos que permite adaptarse al entorno de una organización. Dentro de las capacidades dinámicas, la prospectiva tecnológica se puede clasificar como una capacidad para detectar oportunidades. Por lo tanto, la prospectiva tecnológica es una capacidad dinámica que por medio de la seleccionar, filtrar, analizar e interpretar puede transformar la información del entorno en desarrollos tecnológicos o de investigación.

El presente trabajo aporta al tema de la prospectiva tecnológica al estudiarla desde el enfoque basado en recursos (RBV) en donde se ve a la prospectiva tecnológica como una capacidad de la organización para poder adaptarse a un entorno dinámico y de esta forma ayudar a su supervivencia.

Se sugiere para futuras investigaciones realizar una investigación similar donde el caso de estudio no sea una organización cuya actividad principal sea la investigación y el desarrollo ya que en organizaciones de este tipo tienden a conocer las tendencias futuras en una activad esencial.

\section{Referencias}

Barney, J. (1991). Firm Resources and Sustained Competitive Advantage. Journal of Management, 17(1). doi:10.1177/014920639101700108

Becker, P. (2002). Corporate Foresight in Europe. Retrieved from http://www.foresightplatform.eu/wp-content/uploads/2011/04/EFMN-Brief-No.-82-Corporate-Foresight-inEurope.pdf

Blind, K., Cuhls, K., \& Grupp, H. (1999). Current Foresight Activities in Central Europe. Technological Forecasting and Social Change, 60(1), 15-35. doi:10.1016/S00401625(98)00021-3

Bravo, E., \& Herrera, L. (2009, April). Generación de capacidades dinámicas mediante la innovación organizacional: Un múltiple estudio de casos exploratorio. In XIII Congreso de Ingeniería de Organización (pp. 195-205).

Brown, S. L., \& Eisenhardt, K. M. (1997). The Art of Continuous Change: Linking Complexity Theory and Time-Paced Evolution in Relentlessly Shifting Organizations. Administrative Science Quarterly, 42(1), 1-34. doi:10.2307/2393807 
REVISTA INNOVA ITFIP, 8 (1). 48-66. JUN. 2021

Drever, E. (1995). Using Semi-Structured Interviews in Small-Scale Research. A Teacher's Guide. Edinburgh.

Edington, D. W. (2001). Emerging research: a view from one research center. American Journal of Health Promotion, 15(5), 341-349.

Eisenhardt, K. M. (1989). Making Fast Decisions in High-Velocity Environments (Vol. 32).

Eisenhardt, K. M., \& Martin, J. (2000). Dynamic Capabilities: What are they? Strategic Management Journal, 21(10).

Ende, J. V. D., Mulder, K., Knot, M., Moors, E., \& Vergragt, P. (1998). Traditional and Modern Technology Assessment: Toward a Toolkit. Technological Forecasting and Social Change, 58(1-2), 5-21. doi:10.1016/S0040-1625(97)00052-8

Fallon-Byrne, L., \& Harney, B. (2017). Microfoundations of dynamic capabilities for innovation: a review and research agenda. The Irish Journal of Management, 36(1), 21-31.

Horton, A. (1999). A simple guide to successful foresight. Foresight, 1(1), 5-9. doi:10.1108/14636689910802052

MacKay, B., \& McKiernan, P. (2004). The role of hindsight in foresight: refining strategic reasoning. Futures, 36(2), 161-179. doi:10.1016/S0016-3287(03)00147-2

Martin, B. R. (1995). Foresight in science and technology. Technology Analysis \& Strategic Management, 7(2), 139-168. doi:10.1080/09537329508524202

Medina Vásquez, J. E., \& Ortegón, E. (2006). Manual de prospectiva y decisión estratégica: bases teóricas e instrumentos para América Latina y el Caribe: Cepal.

Miles, I. (2010). The development of technology foresight: A review. Technological Forecasting \& Social Change, 77(9), 1448-1456. doi:10.1016/j.techfore.2010.07.016

Miranda Torrez, J. (2015). El modelo de las capacidades dinámicas en las organizaciones. Investigación administrativa, 44(116), 0-0. 
Pineda, L. (2013). PROSPECTIVA ESTRATÉGICA EN LA GESTIÓN DEL CONOCIMIENTO: UNA PROPUESTA PARA LOS GRUPOS DE INVESTIGACIÓN COLOMBIANOS. Investigación y Desarrollo, 21, 237-311.

Reger, G. (2001). Technology Foresight in Companies: From an Indicator to a Network and Process Perspective. Technology Analysis \& Strategic Management, 13(4), 533-553. doi:10.1080/09537320127286

Rohrbeck, R. (2010). Corporate Foresight: Towards a Maturity Model for the Future Orientation of a Firm. Berlín: Springer-Verlag.

Schwarz, J. O., Rohrbeck, R., \& Wach, B. (2019). Corporate foresight as a microfoundation of dynamic capabilities. FUTURES \& FORESIGHT SCIENCE, e28.

Slaughter, R. A. (1998). Futures Studies as an Intellectual and Applied Discipline. Futures Studies as an Intellectual and Applied Discipline, 42(3), 372-385. doi: $10.1177 / 0002764298042003008$

Teece, D. J. (2007). EXPLICATING DYNAMIC CAPABILITIES: THE NATURE AND MICROFOUNDATIONS OF (SUSTAINABLE) ENTERPRISE PERFORMANCE. Strategic Management Journal, 28(1), 1319-1350. doi:10.1002/smj.640

Teece, D. J. (2017). Business models and dynamic capabilities. Long Range Planning. doi:https://doi.org/10.1016/j.lrp.2017.06.007

Teece, D. J., Pisano, G., \& Shuen, A. (1997). Dynamic Capabilities and Strategic Management. Strategic Management Journal, 18(7), 509-533.

Wernerfelt, B. (1984). A resource-based view of the firm. Strategic Management Journal, 5(2), 171-180. doi:10.1002/smj.4250050207 
Will, M. (2008). Talking about the future within an SME?: Corporate foresight and the potential contributions to sustainable development. Management of Environmental Quality: An International Journal, 19(2), 234-242. doi:doi.org/10.1108/14777830810856618

Zollo, M., \& Winter, S. G. (2002). Deliberate Learning and the Evolution of Dynamic Capabilities. Organization Science, 13(3), 339-351. doi:10.1287/orsc.13.3.339.2780 